\title{
Nonresonant Searches for Axionlike Particles at the LHC
}

\author{
M. B. Gavela, ${ }^{1,2, *}$ J. M. No๑,$^{1,2, \dagger}$ V. Sanz $\oplus^{3, \sharp}$ and J. F. de Trocóniz $\oplus^{1, \S}$ \\ ${ }^{1}$ Departamento de Fisica Teorica, Universidad Autonoma de Madrid, Cantoblanco, 28049 Madrid, Spain \\ ${ }^{2}$ Instituto de Fisica Teorica, IFT-UAM/CSIC, Cantoblanco, 28049 Madrid, Spain \\ ${ }^{3}$ Department of Physics and Astronomy, University of Sussex, BN1 9QH Brighton, United Kingdom
}

(Received 12 September 2019; revised manuscript received 17 December 2019; accepted 6 January 2020; published 6 February 2020)

\begin{abstract}
We propose a new collider probe for axionlike particles (ALPs), and more generally for pseudoGoldstone bosons: nonresonant searches that take advantage of the derivative nature of their interactions with Standard Model particles. ALPs can participate as off shell mediators in the $s$ channel of $2 \rightarrow 2$ scattering processes at colliders like the LHC. We exemplify the power of this novel type of search by deriving new limits on ALP couplings to gauge bosons via the processes $p p \rightarrow Z Z, p p \rightarrow \gamma \gamma$, and $p p \rightarrow j j$ using run 2 CMS public data, probing previously unexplored areas of the ALP parameter space. In addition, we propose future nonresonant searches involving the ALP coupling to other electroweak bosons and/or the Higgs particle.
\end{abstract}

DOI: 10.1103/PhysRevLett.124.051802

Introduction.-Axionlike particles (ALPs) [1,2] and, more generally, pseudo-Goldstone bosons, often appear in extensions of the Standard Model (SM). They may be connected to solutions to the strong $C P$ problem, as in the case of the QCD axion or heavy axions [3-26], and/or to the existence of new spontaneously broken global symmetries in nature. In the following, the term ALP will be used indistinctly to denote all such pseudoscalars.

ALPs are being searched at high-energy colliders [27-37], beam dump experiments [38,39], via their effects in flavor physics [40-45], and through their astrophysical signatures [46-49] (see Ref. [50] for a review).

In this Letter, we propose a novel approach to probe the existence of ALPs at high-energy colliders, namely, nonresonant searches where the ALP is an off shell mediator in the $s$ channel of $2 \rightarrow 2$ scattering processes. The ALP pseudo-Goldstone nature implies that its interactions with SM particles are dominantly derivative, enhancing the cross sections for center-of-mass energies $\hat{s} \gg m_{a}^{2}$, where $m_{a}$ denotes the mass of the ALP $a$. In this kinematical regime, the processes tailored to search for ALPs at the Large Hadron Collider (LHC) include those with two SM bosons in the final state: electroweak gauge bosons $(W, Z, \gamma)$, gluons $g$, and/or the Higgs particle $h$. For $m_{a} \ll 100 \mathrm{GeV}$, the gluon-initiated $2 \rightarrow 2$ diboson scattering processes $p p(g g) \rightarrow Z Z, W W, Z \gamma$,

Published by the American Physical Society under the terms of the Creative Commons Attribution 4.0 International license. Further distribution of this work must maintain attribution to the author(s) and the published article's title, journal citation, and DOI. Funded by SCOAP. and $Z h$ may be mediated by a virtual ALP, as shown in Fig. 1. This can also occur for the processes $p p(g g) \rightarrow$ $j j(g g)$ (with $j$ being hadronic jets) or $p p(g g) \rightarrow \gamma \gamma$ when a large invariant mass $m_{j j}$ or $m_{\gamma \gamma}$ is required in the final state.

The theoretical framework used throughout this Letter is the model-independent approach of effective field theories (EFTs). If the Higgs particle is considered to be part of an exact $\mathrm{SU}(2)_{L}$ doublet at low energies, as predicted in the SM, the putative beyond the Standard Model (BSM) electroweak physics may then be described by an EFT linear expansion [51,52] in terms of towers of gaugeinvariant operators ordered by their mass dimension. Alternatively, since a nondoublet component of the Higgs particle is at present experimentally allowed (at the $\sim 10 \%$ level [53]), a nonlinear EFT (also called chiral) [54-60] based on a momentum expansion is also of interest. In the following, we concentrate on the linear EFT for the SM plus an ALP [1,2,31] and discuss, when pertinent, the comparison with a chiral EFT, notably for the interactions between the ALP and the Higgs boson [31,32].

Bosonic ALP Lagrangian.-Linear expansion: In the linear ALP EFT, the new physics scale to be considered is the ALP decay constant $f_{a}$, which will weight down the higher-dimensional operators built from the SM fields and $a$. The most general $C P$-conserving effective Lagrangian describing bosonic ALP couplings contains - up to next-toleading order (NLO) in a $1 / f_{a}$ expansion-only four independent operators of mass dimension five $[1,2,31,61]$,

$$
\delta \mathcal{L}_{\text {eff }} \supset c_{\tilde{G}} \mathcal{O}_{\tilde{G}}+c_{\tilde{B}} \mathcal{O}_{\tilde{B}}+c_{\tilde{W}} \mathcal{O}_{\tilde{W}}+c_{a \Phi} \mathcal{O}_{a \Phi},
$$

where 

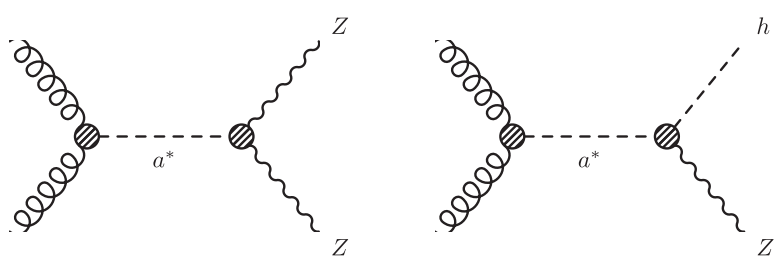

$h$

FIG. 1. Feynman diagrams for the processes $g g \rightarrow Z Z$ (left) and $g g \rightarrow Z h$ (right) via an off shell ALP in the $s$ channel.

$$
\begin{array}{rlrl}
\mathcal{O}_{\tilde{G}} & \equiv-\frac{a}{f_{a}} G_{\mu \nu} \tilde{G}^{\mu \nu}, & \mathcal{O}_{\tilde{W}} \equiv-\frac{a}{f_{a}} W_{\mu \nu}^{a} \tilde{W}_{a}^{\mu \nu}, \\
\mathcal{O}_{\tilde{B}} \equiv-\frac{a}{f_{a}} B_{\mu \nu} \tilde{B}^{\mu \nu}, & \mathcal{O}_{a \Phi} \equiv i \frac{\partial^{\mu} a}{f_{a}} \Phi^{\dagger} \stackrel{\leftrightarrow}{D}_{\mu} \Phi
\end{array}
$$

In these expressions, $G_{\mu \nu}, W_{\mu \nu}$, and $B_{\mu \nu}$ denote, respectively, the $\mathrm{SU}(3)_{c} \times \mathrm{SU}(2) \times \mathrm{U}(1)$ field strengths, and the dual field strengths are defined as $\tilde{X}^{\mu \nu} \equiv \frac{1}{2} \epsilon^{\mu \nu \rho \sigma} X_{\rho \sigma}$, with $\varepsilon^{0123}=1$. The $c_{i}$ constants are real operator coefficients and $\Phi$ denotes the SM Higgs doublet, with $\Phi \stackrel{\leftrightarrow}{D}_{\mu} \Phi \equiv \Phi^{\dagger}\left(D_{\mu} \Phi\right)-\left(D_{\mu} \Phi\right)^{\dagger} \Phi$. The first three operators in Eq. (1) induce physical $a g g, a \gamma \gamma$, $a \gamma Z, a Z Z$, and $a W^{+} W^{-}$interactions,

$$
\begin{aligned}
\delta \mathcal{L}_{\text {eff }} \supset & -\frac{g_{a g g}}{4} a G_{\mu \nu} \tilde{G}^{\mu \nu}-\frac{g_{a \gamma \gamma}}{4} a F_{\mu \nu} \tilde{F}^{\mu \nu}-\frac{g_{a Z \gamma}}{4} a F_{\mu \nu} \tilde{Z}^{\mu \nu} \\
& -\frac{g_{a Z Z}}{4} a Z_{\mu \nu} \tilde{Z}^{\mu \nu}-\frac{g_{a W W}}{4} a W_{\mu \nu} \tilde{W}^{\mu \nu},
\end{aligned}
$$

where

$$
\begin{gathered}
g_{\text {agg }}=\frac{4}{f_{a}} c_{\tilde{G}}, \quad g_{a \gamma \gamma}=\frac{4}{f_{a}}\left(s_{w}^{2} c_{\tilde{W}}+c_{w}^{2} c_{\tilde{B}}\right), \\
g_{a W W}=\frac{4}{f_{a}} c_{\tilde{W}}, \quad g_{a Z Z}=\frac{4}{f_{a}}\left(c_{w}^{2} c_{\tilde{W}}+s_{w}^{2} c_{\tilde{B}}\right), \\
g_{a \gamma Z}=\frac{8}{f_{a}} s_{w} c_{w}\left(c_{\tilde{W}}-c_{\tilde{B}}\right),
\end{gathered}
$$

and $s_{w}$ and $c_{w}$ denote, respectively, the sine and cosine of the Weinberg mixing angle. The Feynman rule for the interaction $a V_{1} V_{2}$ (with $V_{1,2}$ being SM gauge bosons) stemming from these operators is given by

$$
-i g_{a V_{1} V_{2}} p_{V_{1}}^{\rho} p_{V_{2}}^{\sigma} \epsilon_{\mu \nu \rho \sigma} .
$$

The last operator in Eq. (3), $\mathcal{O}_{a \Phi}$, induces a mixing between $a$ and the would-be Goldstone boson eaten by the $Z$. Its physical impact is best illustrated via a Higgs field redefinition, $\Phi \rightarrow \Phi e^{i c_{a \Phi} a / f_{a}}$ [1], which trades $\mathcal{O}_{a \Phi}$ for

$$
i \frac{a}{f_{a}}\left[\bar{Q} Y_{u} \tilde{\Phi} u_{R}-\bar{Q} Y_{d} \Phi d_{R}-\bar{L} Y_{\ell} \Phi \ell_{R}\right]+\text { H.c. },
$$

where $Y_{u, d, \ell}$ denote the SM Yukawa matrices. We focus in this Letter on experimental signals involving ALPs and SM bosons $(W, Z, \gamma, g$, and $h$ ), yet we comment later on signatures involving the $\mathcal{O}_{a \Phi}$ fermionic coupling at the end of the section on nonresonant LHC searches [62].

Chiral expansion: The operators $\mathcal{O}_{\tilde{G}}, \mathcal{O}_{\tilde{W}}$, and $\mathcal{O}_{\tilde{B}}$ in Eq. (3) also appear in the chiral expansion at NLO. In addition, and at variance with the linear EFT, novel ALPHiggs couplings are present in the chiral expansion already at leading order (LO), namely, the operator $\mathcal{A}_{2 D}(h)$ [31], which is a custodial breaking two-derivative operator with mass dimension three,

$\mathcal{L}_{a}^{\mathrm{LO}} \supset c_{2 D} \mathcal{A}_{2 D}(h)=c_{2 D}\left[i v^{2} \operatorname{Tr}\left[\mathbf{T V}_{\mu}\right] \partial^{\mu} \frac{a}{f_{a}} \mathcal{F}(h)\right]$,

where $v=246 \mathrm{GeV}$ denotes the electroweak scale as defined from the $W$ mass, $\mathbf{V}_{\mu}(x) \equiv\left[\mathbf{D}_{\mu} \mathbf{U}(x)\right] \mathbf{U}(x)^{\dagger}, \mathbf{T}(x) \equiv$ $\mathbf{U}(x) \sigma_{3} \mathbf{U}(x)^{\dagger}$, and $\mathbf{U}(x)=e^{i \sigma_{j} \pi^{j}(x) / v}$, with $\pi^{j}(x)$ corresponding to the longitudinal degrees of freedom of the electroweak gauge bosons, and $\sigma_{j}$ are the Pauli matrices. The physical Higgs particle $h$ is introduced in the chiral expansion via polynomial functions [63] of $h / v, \mathcal{F}(h)=$ $1+a_{2 D} h / v+b_{2 D}(h / v)^{2}+\mathcal{O}\left(h^{3} / v^{3}\right)$, with $a_{2 D}, b_{2 D}$ as constant coefficients. $\mathcal{A}_{2 D}$ is the chiral counterpart ("sibling") of the linear operator $\mathcal{O}_{a \Phi}$ in Eq. (3), with a key difference: in addition to ALP-fermion couplings analogous to those in Eq. (8), $\mathcal{A}_{2 D}$ induces interactions between the ALP, the electroweak gauge bosons, and any number of Higgs particles, e.g., a trilinear $a-Z-h$ coupling (see Fig. 1). The associated experimental signatures at the LHC will be discussed at the end of the section on nonresonant LHC searches. Note that such couplings can be found in the linear expansion only at next-to-NLO, i.e., mass dimension seven [32,64], and are thus expected to yield subleading effects in that case.

Nonresonant ALP-mediated diboson production.-The key observation is that, due to the derivative nature of the ALP interactions under discussion, the ALP-mediated scattering processes $g g \rightarrow a^{*} \rightarrow V_{1} V_{2}$ exhibit a harder scaling with the invariant mass of the event $\sqrt{\hat{s}}=m_{V_{1} V_{2}}$ than usual $s$-channel-mediated exchanges.

In all generality, the contributions from bosonic ALP couplings in Eq. (3) interfere with the absorptive part of SM $2 \rightarrow 2$ diboson amplitudes. Nevertheless, given the present loose bounds on ALP couplings (see, e.g., [65] for details), pure ALP exchange dominates the cross section for some LHC channels. A quartic dependence on ALP couplings results in this case,

$$
\sigma_{V_{1} V_{2}} \propto g_{a g g}^{2} g_{a V_{1} V_{2}}^{2} \hat{s} \sim \frac{\hat{s}}{f_{a}^{4}},
$$

in the ALP off shell regime $\hat{s} \gg m_{a}^{2}, m_{V_{i}}^{2}$. (This has been noted in a different setup in Ref. [34].) The same type of 
energy behavior holds for $g g \rightarrow a^{*} \rightarrow Z h$ from Eq. (9). Such energy dependence is valid only as far as the energies probed in the scattering process are smaller than the cutoff scale of the EFT, $\sqrt{\hat{s}}<f_{a}$.

The behavior in Eq. (10) is to be compared with the energy dependence for a usual $2 \rightarrow 2 s$-channel-mediated process, which scales instead as $1 / \hat{s}$ far above from the $s$ channel resonance. Factoring in the proton parton distribution functions, which tame the energy growth in Eq. (10), the differential cross section for the ALP-mediated process $p p \rightarrow a^{*} \rightarrow V_{1} V_{2}$ diminishes-at energies much larger than the resonance's mass-more slowly with the invariant mass than for a usual $s$-channel resonance whose couplings do not depend on the momenta involved. The momentum dependence of the ALP interaction in Eq. (7) significantly smooths out the decrease of the cross section at large $\sqrt{\hat{s}}$, allowing us to distinguish ALP-mediated processes from the SM background.

For sufficiently small ALP couplings, the size of the interference with the SM background becomes comparable to the pure ALP signal and must be taken into account. The value of the couplings for which this happens depends on the specific final state $V_{1} V_{2}$. For $\gamma \gamma$ and the other electroweak diboson final states, the ALP signal interferes with one-loop $\mathrm{SM}$ processes. The interference is constructive or destructive depending on the relative sign of $g_{a g g}$ and $g_{a V_{1} V_{2}}$; in any case, it can be disregarded at present, given the size of ALP couplings that can be currently probed at the LHC (see section on nonresonant LHC searches). Future LHC analyses will need to include them, though, and this exploration is deferred to a future work. The situation is different for dijet ( $g$ g) final states: the SM contributes at tree level (via gluon exchange), and the interference, always destructive in this case, already dominates at present over the pure ALP signal for the $g_{\text {agg }}$ values at reach. An analysis of $g g \rightarrow g g$, where the effect of interference is taken into account, is presented in the Supplemental Material [66].

The nonresonant $s$-channel ALP signatures explored in this Letter have several further attractive features: (i) In the regime under discussion with $\hat{s} \gg m_{a}^{2}$, the signal cross section and distributions are essentially independent of the value of $m_{a}$. This implies that the search is equally sensitive to any $m_{a}$ significantly below the energy range probed by the search. In particular, for the LHC searches considered in the next section, the derived sensitivity can be safely applied to any ALP mass below $100 \mathrm{GeV}$. (ii) Being a nonresonant process, no hypothesis is needed on the value of other possible couplings that do not contribute to the considered process. This is at variance with on shell analyses, for which the dependence on other ALP couplings may appear through the partial decay widths [71]. In this sense, nonresonant searches are more model independent and thus more robust.

From a theoretical point of view, the $g_{a V_{1} V_{2}}$ couplings depend only on the ratio $c_{i} / f_{a}$ [see Eqs. (4)-(6)], but the value of $f_{a}$ is relevant to assess the validity of the EFT, which limits the energy range that can be safely considered in a LHC search (e.g., to bins satisfying $\sqrt{\hat{s}}<f_{a}$ ) [72], and we discuss this for specific LHC searches in the next section. Another pertinent question is the possible impact of radiative corrections and of higher-dimensional operators. For the former, self-energy corrections to the $s$ channel ALP propagator only become non-negligible close to the EFT validity boundary, and we do not consider their effect here. Higher-dimensional operators, e.g., those weighed down by the same $\mathcal{O}\left(1 / f_{a}^{2}\right)$ factor as the amplitudes discussed above, can also contribute only at loop level, as $f_{a}$ must intervene as powers of $a / f_{a}$ and no ALP is present in the final states considered here. Furthermore, only by engineered fine-tuned cancellations could such operators impact significantly the results of this Letter.

Nonresonant LHC searches.-In this section, we derive new limits on $g_{a V_{1} V_{2}}$ couplings through the nonresonant ALP-mediated processes discussed above, using public data from LHC run $2(\sqrt{s}=13 \mathrm{TeV})$ CMS searches. ALP production in the $s$ channel is dominated by gluon-gluon fusion, as the $q \bar{q}$ induced ALP production amplitude is proportional to the quark masses (see [62]) and thus highly suppressed. Possible final states to be considered include $g g, Z Z, W W, Z \gamma, \gamma \gamma$, or $Z h$. While it is of high interest to explore all of them, since they probe different operator combinations within the EFT, we focus below on the processes $p p \rightarrow a^{*} \rightarrow Z Z, p p \rightarrow a^{*} \rightarrow \gamma \gamma$, and $p p \rightarrow a^{*} \rightarrow g g$. For these channels, the CMS collaboration has recently published new results, providing explicit calculations of the corresponding SM backgrounds. We use those public data to compute approximate limits on

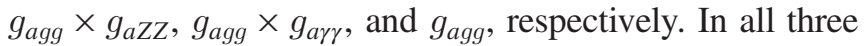
analyses, the ALP mass is fixed to $m_{a}=1 \mathrm{MeV}$ (i.e., effectively massless at LHC energies) and the ALP width $\Gamma_{a}$ is assumed to respect $\Gamma_{a} \ll m_{a}$.

For the $p p \rightarrow a^{*} \rightarrow Z Z$ and $p p \rightarrow a^{*} \rightarrow \gamma \gamma$ channels, our sensitivities are estimated from a simplified binned likelihood ratio analysis. The likelihood function is built as a product of bin Poisson probabilities

$$
L(\mu)=\prod_{k} e^{-\left(\mu s_{k}+b_{k}\right)}\left(\mu s_{k}+b_{k}\right)^{n_{k}} / n_{k} !,
$$

where $n_{k}, b_{k}$, and $s_{k}$ denote, respectively, the observed data, SM background, and ALP signal prediction in a given bin $k$, and the signal strength modifier $\mu$ is taken as the only floating parameter in the likelihood fit (see Ref. [31] for details). No systematic uncertainties are considered, for simplicity, since SM background statistics is largely the dominant source of uncertainty in these analyses. For the $p p \rightarrow a^{*} \rightarrow g g$ channel, we perform a $\chi^{2}$ fit to the data, including systematic errors but no bin-to-bin correlations.

Other important search channels are also briefly discussed below, albeit their analysis is left for the future: 
$p p \rightarrow a^{*} \rightarrow Z \gamma, p p \rightarrow a^{*} \rightarrow Z h$ [which provides a unique window into the chiral EFT via the operator $\mathcal{A}_{2 D}$ in Eq. (9)], and $p p \rightarrow a^{*} \rightarrow t \bar{t}$ [which would yield access to the operator $\mathcal{O}_{a \Phi}$ in Eq. (3)].

$p p \rightarrow a^{*} \rightarrow Z Z$ : The process $p p \rightarrow a^{*} \rightarrow Z Z \rightarrow \ell \ell q \bar{q}$ is studied next, following the semileptonic diboson CMS analysis at LHC $\sqrt{s}=13 \mathrm{TeV}$ with $35.9 \mathrm{fb}^{-1}$ [73]. We focus on the "low-mass merged" CMS analysis category targeting the invariant mass region $m_{Z Z} \in[450,2000] \mathrm{GeV}$, with one $Z$ boson decaying leptonically, $Z \rightarrow \ell \ell$, and the other decaying hadronically. The boosted hadronic $Z$ decay products are required to merge into a single jet, $Z \rightarrow J$. The jet is reconstructed via the anti- $k_{T}$ algorithm with $R=0.8$. Our signal process is simulated in MadGraph5_aMC@NLO [74], with a subsequent parton showering and hadronization with Pythia 8 [75] and detector simulation via Delphes 3 [76], including the use of jet-substructure variables as discussed in the Supplemental Material [66]. Following Ref. [73], the analysis is divided into b-tagged and untagged categories, targeting, respectively, the $Z \rightarrow b \bar{b}$ and $Z \rightarrow q \bar{q}$ (with $q=u, d, s, c$ ) decays. The $b$ tagging of the merged jet $J$ provides a strong background suppression, yielding a further increase in sensitivity.

As an illustration of the impact of the derivative nature of ALP interactions, the $\sqrt{s}=13 \mathrm{TeV}$ cross section $\sigma(p p \rightarrow$ $\left.a^{*} \rightarrow Z Z\right)$ for $c_{\tilde{G}}=c_{\tilde{W}}=c_{\tilde{B}}=1$ and $f_{a}=1 \mathrm{TeV}$ is $81 \mathrm{pb}$. The CMS event selection is discussed in detail in the Supplemental Material [66]. Figure 2 shows the invariant mass $m_{\ell \ell J}$ distribution resulting for the signal after the CMS event selection, for $c_{i}=1$ and $f_{a}=2 \mathrm{TeV}$ (corresponding to the largest value of $m_{\ell \ell J}$ in the CMS analysis), together with the SM background publicly available in Ref. [73] (and dominated by $Z+$ jets), both for the untagged (top plot) and $b$-tagged (bottom plot) categories. A binned likelihood analysis of the $m_{\ell \ell J}$ distribution after CMS event selection combining the untagged and $b$-tagged categories is then performed, which allows us to set a $95 \%$ C.L. exclusion limit on the signal cross section of $\sigma=25 \mathrm{fb}$. This corresponds to $f_{a}>4.1 \mathrm{TeV}$ for $c_{i}=1$ and is valid for any value of the ALP mass up to $m_{a} \sim 200 \mathrm{GeV}$ without significant modifications of the signal properties. Note that, since the lowmass merged CMS analysis uses data up to $m_{Z Z}=2 \mathrm{TeV}$, our derived limit on $f_{a}$ for $c_{i}=1$ lies within the region of validity of the EFT. In Fig. 3 (top), the corresponding new limit on $g_{a z Z}$ [see Eq. (5)] resulting from our nonresonant analysis is depicted as a hatched area, for a fixed value $g_{\text {agg }}^{-1}=1 \mathrm{TeV}$.

For comparison, Fig. 3 (top) depicts previous bounds in the literature for $g_{a z z}$ as well, which also assume the additional presence of $g_{\text {agg }}$, albeit obtained from on shell ALP searches. For $m_{a} \lesssim 0.1 \mathrm{GeV}$, the ALP is stable on LHC scales, resulting in constraints on $g_{a z Z}$ from mono- $Z$ searches (in violet), see Ref. [31]. A nonvanishing $g_{a z Z}$ yields a radiative (two-loop) contribution to the ALP
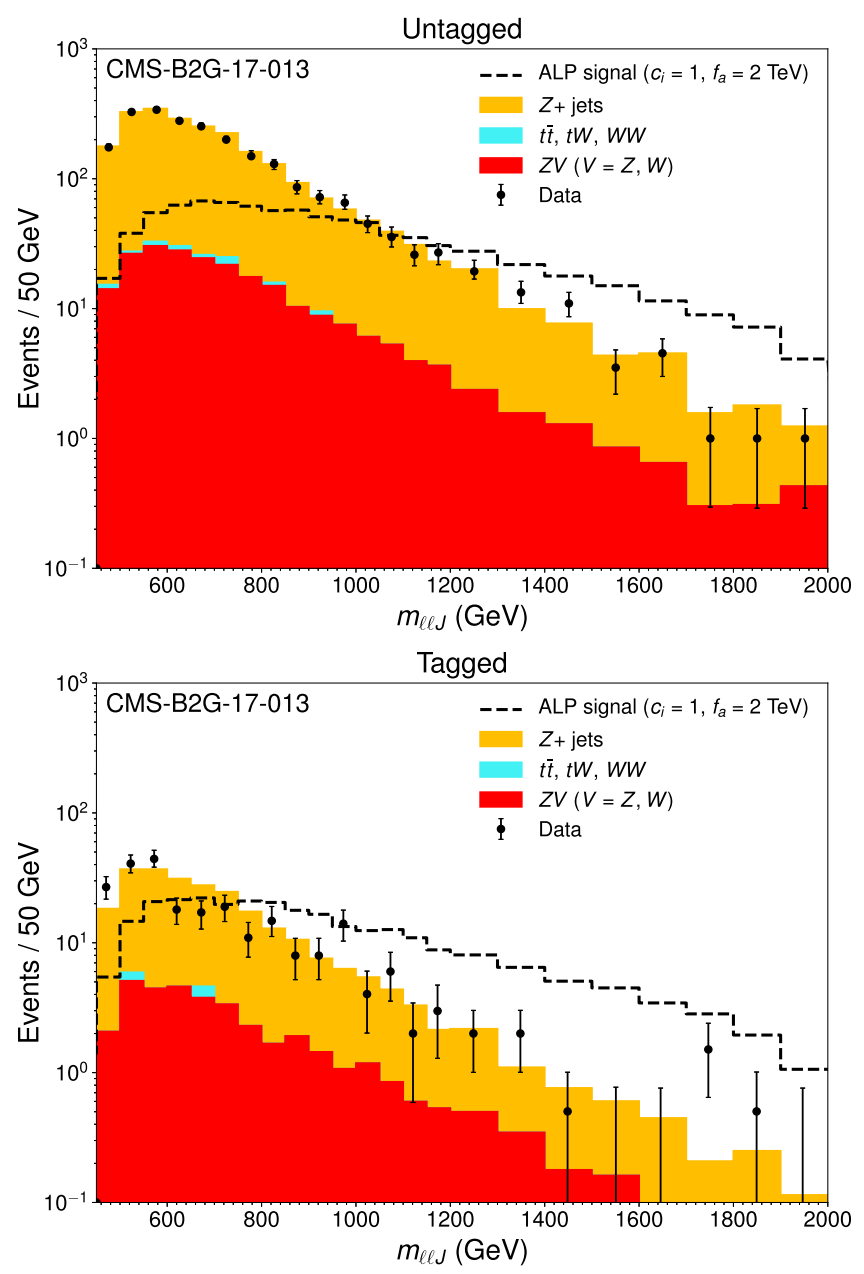

FIG. 2. $m_{\ell \ell J}$ distributions for the ALP ZZ signal with $c_{i}=1$, $f_{a}=2 \mathrm{TeV}$ (dashed black line), and SM background from $Z+$ jets (yellow), $Z V$ (red), and $t \bar{t}$ (cyan) after CMS event selection, in the untagged (top) and $b$-tagged (bottom) categories. The experimental data are shown as black dots.

coupling to photons $g_{a \gamma \gamma}$, which allows us to obtain further constraints on $g_{a Z Z}$ for certain ranges of ALP masses for which strong constraints on $g_{a y \gamma}$ exist (see the discussion in Refs. [32,65]). For ALP masses below the GeV scale, limits on $g_{a z Z}$ are thus set by beam dump searches (in yellow) [79-81] (we adapt here the bounds compiled in Ref. [38]) and by energy-loss arguments applied to the supernova SN1987a [48,49] (in blue), both through absence of extra cooling (labeled "length" in Fig. 3 and through the absence of a photon burst from decaying emitted axions (labeled "decay" in Fig. 3). Furthermore, the radiative contribution of $g_{a Z Z}$ to $g_{a \gamma \gamma}$ is also constrained by LHCb [82] (see Ref. [42]) in the small region $4.9<m_{a}<6.3 \mathrm{GeV}$ (in dark gray) and by ATLAS and CMS searches for $\gamma \gamma$ resonances (in red) for $m_{a}>10 \mathrm{GeV}$ (we show the bounds from Refs. [27,33] using the experimental data from [83-88], as compiled in [65]). We stress that the latter limits are from LHC run $1(\sqrt{s}=7$ and $8 \mathrm{TeV})$, and as such $\sqrt{s}=13 \mathrm{TeV}$ 


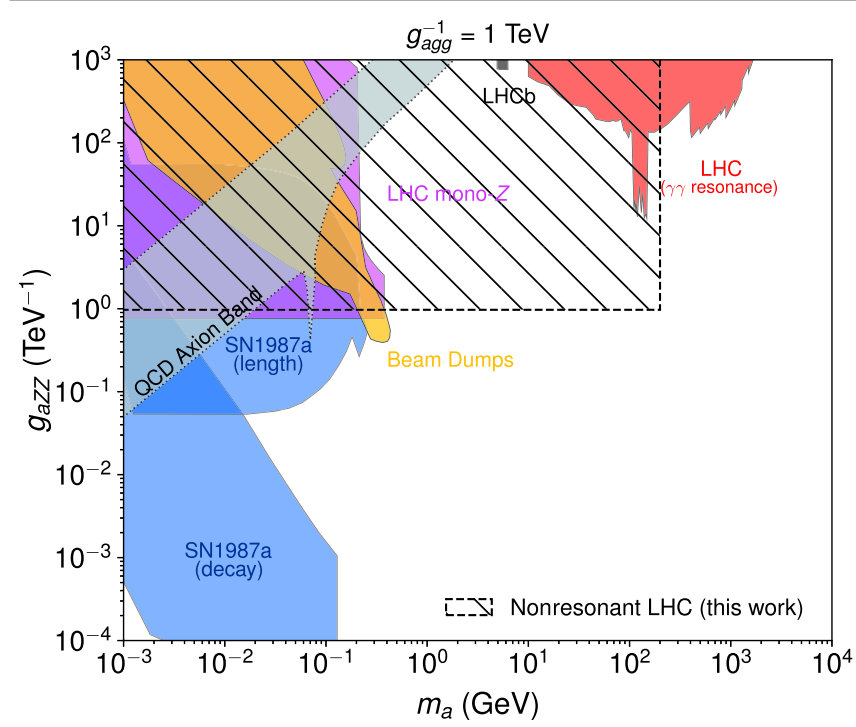

the theoretical band corresponding to the QCD axion that solves the strong $C P$ problem is shown (in light gray) for completeness, as taken from Ref. [65] for updated estimations of the Kim-Shifman-Vainshtein-Zakharov axion model [89].

pp $\rightarrow \mathbf{a}^{*} \rightarrow \gamma \gamma$ : Nonresonant ALP searches are also possible for final states to which a light ALP could decay, such as $\gamma \gamma$, by selecting events with a large invariant mass $m_{\gamma \gamma} \gg m_{a}$. The recent CMS search for nonresonant new physics in $\gamma \gamma$ final states [77] with $35.9 \mathrm{fb}^{-1}$ of $13 \mathrm{TeV}$ LHC data is used here. In analogy with the previous section, we simulate the signal process $p p \rightarrow a^{*} \rightarrow \gamma \gamma$ with MadGraph5_aMC@NLO, Pythia 8, and Delphes 3, obtaining a signal cross section $\sigma\left(p p \rightarrow a^{*} \rightarrow \gamma \gamma\right)=47 \mathrm{pb}$ for $c_{\tilde{G}}=$ $c_{\tilde{W}}=c_{\tilde{B}}=1, f_{a}=1 \mathrm{TeV}$, and with the initial requirement $m_{\gamma \gamma}>500 \mathrm{GeV}$. The subsequent CMS event selection applied here is detailed in the Supplemental Material [66], with the main SM backgrounds [77] being $\gamma \gamma$ and $\gamma+j$ (with the jet $j$ misidentified as a photon). After the event selection, we perform a binned likelihood analysis of the $m_{\gamma \gamma}$ distribution for the two selection categories discussed in the Supplemental Material [66] according to the rapidity of the photons. This leads to a combined 95\% C.L. observed exclusion limit on the signal cross section of $\sigma \simeq 1.2 \mathrm{fb}$. This limit corresponds to $f_{a}>$ 14.2 TeV for $c_{i}=1$, which we find to be valid up to $m_{a} \sim$ $200 \mathrm{GeV}$ without significant modifications of the signal properties. The resulting bound on $g_{a \gamma \gamma}$ is depicted in Fig. 3 (bottom) for $g_{\text {agg }}^{-1}=1 \mathrm{TeV}$ as a hatched area. Bounds from resonant searches at the LHC, beam dump experiments, and astrophysical constraints (supernova SN1987a) are also shown, see last section for details. For comparison, the figure also shows bounds from resonant searches by $B A B A R$ [90] (in dark gray) (as obtained from Ref. [42]), from L3 [78] (in cyan), and from large electron positron (LEP) collider searches (in green) for new physics in $e^{+} e^{-} \rightarrow 2 \gamma, 3 \gamma$ processes (see Refs. [28,29] for a detailed discussion). Regarding the latter, Refs. [28,29] assume a vanishing gluonic coupling $g_{\text {agg }}$ and thus apply in our case only for $m_{a}<3 m_{\pi}$; for $m_{a}>3 m_{\pi}$ the ALP can decay into hadronic final states in the presence of a nonvanishing $g_{\text {agg }}$ coupling, which could significantly weaken the LEP bounds, and we refrain to claim an exclusion in that region. We also do not include here projected limits on $g_{\text {ary }}$ from light-by-light scattering at the LHC in proton-proton [34] and $P b-P b$ collisions [30] (the latter is also significantly weakened in the present scenario by the presence of $g_{a g g}$ ), as they are not competitive with the search presented here.

Further nonresonant ALP searches: (i) $p p \rightarrow a^{*} \rightarrow g g$. As discussed previously, the gluonic coupling $g_{a g g}$ can be constrained independently, using dijet searches at the LHC. We have used the $13 \mathrm{TeV}$ CMS search on dijet angular distributions [91] for this purpose, with the details 
of our analysis given in the Supplemental Material [66]. A $95 \%$ C.L. excluded region of $1.9<f_{a}<3 \mathrm{TeV}$ for $c_{\tilde{G}}=1$ follows, including the interference between the ALP signal and the SM background, which dominates over the pure ALP signal in the region considered. This bound is to be taken only as a qualitative estimate, as the analysis uses data in the 2.4-3 TeV range and thus in the limit of validity of the EFT. Note also that the limit obtained is weaker than the benchmark value $g_{a g g}^{-1}=1 \mathrm{TeV}$ used in Fig. 3 (corresponding to $f_{a} / c_{\tilde{G}}=4 \mathrm{TeV}$ ), as it should be.

(ii) $p p \rightarrow a^{*} \rightarrow Z h$. This process yields a powerful probe of the chiral EFT through the operator $\mathcal{A}_{2 D}$ in Eq. (9). For $Z \rightarrow \ell \ell$ and $h \rightarrow \bar{b} b$ this signature is similar to that analyzed in the last section for the $b$-tagged category, since the process $p p \rightarrow a^{*} \rightarrow Z h$ has similar $m_{\ell \ell J}$ kinematics and expected cross section than $p p \rightarrow a^{*} \rightarrow Z Z$, for $c_{2 D} \simeq c_{\tilde{W}}, c_{\tilde{B}}$. This suggests that the analysis performed above could be adapted to probe very efficiently the ALPmediated $Z h$ signal. Furthermore, there are several advantages in performing a dedicated $Z h$ search along the lines in Ref. [73]: the SM background distribution for the merged jet mass $m_{J}$ is smaller around $m_{h}$ than around $m_{Z}$ (as shown in Ref. [73]), and the SM backgrounds after the CMS event selection are significantly smaller in the $b$-tagged category, as shown in Fig. $2 ; h$ decays dominantly to $\bar{b} b$.

(iii) $p p \rightarrow a^{*} \rightarrow t \bar{t}$. This channel allows us to probe the ALP-fermion couplings induced by the operator $\mathcal{O}_{a \Phi}$ in the ALP linear EFT. Because the amplitude of any physical ALP-fermion coupling is proportional to the fermion Yukawa couplings [see Eq. (8) and [62] ], ALP production via gluon fusion with $t \bar{t}$ in the final state is an optimal channel that deserves detailed future study [92].

(iv) $p p \rightarrow a^{*} \rightarrow Z \gamma$. This channel provides a key complementary probe to the $Z Z$ and $\gamma \gamma$ searches discussed in the first two parts of this section, given its clean signature. The nonresonant analysis of this channel using public information (e.g., from Ref. [93]) requires, however, further assumptions with respect to the $Z Z$ and $\gamma \gamma$ analyses. The study of ALP-mediated $Z \gamma$ signatures is thus left for a forthcoming work.

Conclusions and outlook.-In this Letter, we have proposed a new approach to probe the existence of ALPs (and, more generally, of pseudo-Goldstone bosons) via nonresonant searches at the LHC where the ALP can be produced as an $s$-channel off shell mediator. The search takes advantage of the derivative nature of the ALP interactions with SM particles. Using CMS $13 \mathrm{TeV}$ public data, we have derived new limits on ALP couplings to SM gauge bosons via the processes $p p \rightarrow Z Z, p p \rightarrow \gamma \gamma$, and $p p \rightarrow j j(g g)$. These provide the most stringent bounds on ALPs over a wide region of masses, in the presence of an ALP-gluonic coupling $g_{\text {agg }}$. They have the advantage of being equally sensitive to light ALPs with masses up to the kinematical energy scale of the LHC analyses considered $\sim \mathcal{O}(100) \mathrm{GeV}$. Possible extensions of the analysis to other final states such as $Z \gamma, Z h$ and fermionic final states $(t \bar{t})$ have been discussed as well, altogether highlighting the power of nonresonant searches for ALPs at colliders.

We thank Gonzalo Alonso-Alvarez, Jesús Bonilla, Pablo Quilez, and Stefan Pokorsky for useful discussions and comments on this Letter. The work of V. S. is funded by the Science Technology and Facilities Council (STFC) under Grant No. ST/P000819/1. M. B. G. and J. F. T. acknowledge support from the "Spanish Agencia Estatal de Investigación" (AEI) and the EU "Fondo Europeo de Desarrollo Regional" (FEDER) through the Projects FPA2016-78645-P and FPA2017-84260-C3-2-R, respectively. The work of J. M. N. was supported by the Programa Atracción de Talento de la Comunidad de Madrid via Grant No. 2017-T1/TIC-5202 and by Grant No. PGC2018096646-A-I00 from the Spanish Proyectos de I + D de Generación de Conocimiento. M. B. G. and J.M. N. acknowledge support from the European Union's Horizon 2020 Research and Innovation Programme under the Marie Sklodowska-Curie Grants No. 690575 (Research and Innovation Staff Exchange InvisiblesPlus) and No. 674896 (Initial Training Network ELUSIVES), as well as from the Spanish Research Agency (Agencia Estatal de Investigación) through the Grant Instituto de Fisica Teorica Centro de Excelencia Severo Ochoa SEV2016-0597. In addition, they warmly thank Venus Keus for hospitality at the University of Helsinki during the very last stages of this work.

\footnotetext{
*belen.gavela@uam.es

josemiguel.no@uam.es

*v.sanz@sussex.ac.uk

§orge.troconiz@uam.es
}

[1] H. Georgi, D. B. Kaplan, and L. Randall, Phys. Lett. 169B, 73 (1986).

[2] K. Choi, K. Kang, and J. E. Kim, Phys. Lett. B 181, 145 (1986).

[3] R. D. Peccei and H. R. Quinn, Phys. Rev. Lett. 38, 1440 (1977).

[4] R. D. Peccei and H. R. Quinn, Phys. Rev. D 16, 1791 (1977).

[5] F. Wilczek, Phys. Rev. Lett. 40, 279 (1978).

[6] S. Weinberg, Phys. Rev. Lett. 40, 223 (1978).

[7] L. Susskind, Phys. Rev. D 20, 2619 (1979).

[8] M. A. Shifman, A. I. Vainshtein, and V. I. Zakharov, Nucl. Phys. B166, 493 (1980).

[9] J. E. Kim, Phys. Rev. Lett. 43, 103 (1979).

[10] A. R. Zhitnitsky, Yad. Fiz. 31, 497 (1980) [Sov. J. Nucl. Phys. 31, 260 (1980)].

[11] M. Dine, W. Fischler, and M. Srednicki, Phys. Lett. 104B, 199 (1981).

[12] S. Dimopoulos and J. Preskill, Nucl. Phys. B199, 206 (1982).

[13] K. Choi and J. E. Kim, Phys. Rev. D 32, 1828 (1985).

[14] V. A. Rubakov, JETP Lett. 65, 621 (1997). 
[15] Z. Berezhiani, L. Gianfagna, and M. Giannotti, Phys. Lett. B 500, 286 (2001).

[16] S. D. H. Hsu and F. Sannino, Phys. Lett. B 605, 369 (2005).

[17] A. Hook, Phys. Rev. Lett. 114, 141801 (2015).

[18] H. Fukuda, K. Harigaya, M. Ibe, and T. T. Yanagida, Phys. Rev. D 92, 015021 (2015).

[19] C. W. Chiang, H. Fukuda, M. Ibe, and T. T. Yanagida, Phys. Rev. D 93, 095016 (2016).

[20] T. Gherghetta, N. Nagata, and M. Shifman, Phys. Rev. D 93, 115010 (2016).

[21] S. Dimopoulos, A. Hook, J. Huang, and G. MarquesTavares, J. High Energy Phys. 11 (2016) 052.

[22] A. Kobakhidze, arXiv:1607.06552.

[23] P. Agrawal and K. Howe, J. High Energy Phys. 12 (2018) 029.

[24] P. Agrawal and K. Howe, J. High Energy Phys. 12 (2018) 035.

[25] M. K. Gaillard, M. B. Gavela, R. Houtz, P. Quilez, and R. Del Rey, Eur. Phys. J. C 78, 972 (2018).

[26] M. B. Gavela, M. Ibe, P. Quilez, and T. T. Yanagida, Eur. Phys. J. C 79, 542 (2019).

[27] J. Jaeckel, M. Jankowiak, and M. Spannowsky, Phys. Dark Universe 2, 111 (2013).

[28] K. Mimasu and V. Sanz, J. High Energy Phys. 06 (2015) 173.

[29] J. Jaeckel and M. Spannowsky, Phys. Lett. B 753, 482 (2016).

[30] S. Knapen, T. Lin, H. K. Lou, and T. Melia, Phys. Rev. Lett. 118, 171801 (2017).

[31] I. Brivio, M. B. Gavela, L. Merlo, K. Mimasu, J. M. No, R. del Rey, and V. Sanz, Eur. Phys. J. C 77, 572 (2017).

[32] M. Bauer, M. Neubert, and A. Thamm, J. High Energy Phys. 12 (2017) 044.

[33] A. Mariotti, D. Redigolo, F. Sala, and K. Tobioka, Phys. Lett. B 783, 13 (2018).

[34] C. Baldenegro, S. Fichet, G. von Gersdorff, and C. Royon, J. High Energy Phys. 06 (2018) 131.

[35] N. Craig, A. Hook, and S. Kasko, J. High Energy Phys. 09 (2018) 028.

[36] E. Arganda, A. D. Medina, N. I. Mileo, R. A. Morales, and A. Szynkman, Phys. Lett. B 789, 575 (2019).

[37] J. Ebadi, S. Khatibi, and M. Mohammadi Najafabadi, Phys. Rev. D 100, 015016 (2019).

[38] B. Dobrich, J. Jaeckel, F. Kahlhoefer, A. Ringwald, and K. Schmidt-Hoberg, J. High Energy Phys. 02 (2016) 018.

[39] B. Dobrich, J. Jaeckel, and T. Spadaro, J. High Energy Phys. 05 (2019) 213.

[40] M. B. Gavela, R. Houtz, P. Quilez, R. Del Rey, and O. Sumensari, Eur. Phys. J. C 79, 369 (2019).

[41] E. Izaguirre, T. Lin, and B. Shuve, Phys. Rev. Lett. 118, 111802 (2017).

[42] X. Cid Vidal, A. Mariotti, D. Redigolo, F. Sala, and K. Tobioka, J. High Energy Phys. 01 (2019) 113.

[43] B. Dobrich, F. Ertas, F. Kahlhoefer, and T. Spadaro, Phys. Lett. B 790, 537 (2019).

[44] D. Aloni, Y. Soreq, and M. Williams, Phys. Rev. Lett. 123, 031803 (2019).

[45] L. Merlo, F. Pobbe, S. Rigolin, and O. Sumensari, J. High Energy Phys. 06 (2019) 091.
[46] V. Anastassopoulos et al. (CAST Collaboration), Nat. Phys. 13, 584 (2017).

[47] E. Armengaud et al., J. Instrum. 9, T05002 (2014).

[48] A. Payez, C. Evoli, T. Fischer, M. Giannotti, A. Mirizzi, and A. Ringwald, J. Cosmol. Astropart. Phys. 02 (2015) 006.

[49] J. Jaeckel, P. C. Malta, and J. Redondo, Phys. Rev. D 98, 055032 (2018).

[50] R. Essig et al., arXiv:1311.0029.

[51] W. Buchmuller and D. Wyler, Nucl. Phys. B268, 621 (1986).

[52] B. Grzadkowski, M. Iskrzynski, M. Misiak, and J. Rosiek, J. High Energy Phys. 10 (2010) 085.

[53] G. Aad et al. (ATLAS Collaboration), Phys. Rev. D 101, 012002 (2020).

[54] F. Feruglio, Int. J. Mod. Phys. A 08, 4937 (1993).

[55] R. Alonso, M. B. Gavela, L. Merlo, S. Rigolin, and J. Yepes, J. High Energy Phys. 06 (2012) 076.

[56] A. Azatov, R. Contino, and J. Galloway, J. High Energy Phys. 04 (2012) 127; 04 (2013) 140(E).

[57] R. Alonso, M. B. Gavela, L. Merlo, S. Rigolin, and J. Yepes, Phys. Lett. B 722, 330 (2013); 726, 926(E) (2013).

[58] R. Alonso, M. B. Gavela, L. Merlo, S. Rigolin, and J. Yepes, Phys. Rev. D 87, 055019 (2013).

[59] G. Buchalla, O. Cata, and C. Krause, Nucl. Phys. B880, 552 (2014); B913, 475(E) (2016).

[60] I. Brivio, T. Corbett, O. J. P. Eboli, M. B. Gavela, J. Gonzalez-Fraile, M. C. Gonzalez-Garcia, L. Merlo, and S. Rigolin, J. High Energy Phys. 03 (2014) 024.

[61] A. Salvio, A. Strumia, and W. Xue, J. Cosmol. Astropart. Phys. 01 (2014) 011.

[62] A complete-bosonic and fermionic-ALP basis can be obtained substituting $\mathcal{O}_{a \Phi}$ in Eq. (3) by general flavorchanging fermionic operators, e.g., $[1,2,31,61]$. The physical effects of the latter are proportional to the Yukawa couplings of the fermions involved.

[63] B. Grinstein and M. Trott, Phys. Rev. D 76, 073002 (2007).

[64] M. Bauer, M. Neubert, and A. Thamm, Phys. Rev. Lett. 117, 181801 (2016).

[65] G. Alonso-Alvarez, M. B. Gavela, and P. Quilez, Eur. Phys. J. C 79, 223 (2019).

[66] See Supplemental Material at http://link.aps.org/ supplemental/10.1103/PhysRevLett.124.051802, which includes Refs. [67-70], for details on the analysis including jet reconstruction and the use of jet substructure.

[67] A. Buckley, J. Ferrando, S. Lloyd, K. Nordström, B. Page, M. Rüfenacht, M. Schönherr, and G. Watt, Eur. Phys. J. C 75, 132 (2015).

[68] J. Thaler and K. Van Tilburg, J. High Energy Phys. 03 (2011) 015.

[69] A. M. Sirunyan et al. (CMS Collaboration), J. Instrum. 13, P05011 (2018).

[70] M. Cacciari, G. P. Salam, and G. Soyez, Eur. Phys. J. C 72, 1896 (2012).

[71] Most present ALP limits based on resonant processes have considered only one independent $g_{a V_{1} V_{2}}$ coupling at a time among the set in Eq. (3) (see, e.g., Refs. [28-30,38,41]), with some recent analyses considering the simultaneous presence of two or at most three independent couplings $[33,40,65]$. 
[72] If the underlying BSM theory were in the weak coupling regime and led at one loop to the operators in Eq. (3), their coefficients could plausibly be suppressed by an additional $\alpha_{i} /(8 \pi)$ factor. This would drastically reduce the set of valid energy bins in LHC searches. We stick here instead to the general and widespread definitions in Eqs. (3) and (3).

[73] A. M. Sirunyan et al. (CMS Collaboration), J. High Energy Phys. 09 (2018) 101.

[74] J. Alwall et al., J. High Energy Phys. 07 (2014) 079.

[75] T. Sjöstrand, S. Ask, J. R. Christiansen, R. Corke, N. Desai, P. Ilten, S. Mrenna, S. Prestel, C. O. Rasmussen, and P. Z. Skands, Comput. Phys. Commun. 191 (2015) 159.

[76] J. de Favereau, C. Delaere, P. Demin, A. Giammanco, V. Lemaître, A. Mertens, and M. Selvaggi (DELPHES 3 Collaboration), J. High Energy Phys. 02 (2014) 057.

[77] A. M. Sirunyan et al. (CMS Collaboration), Phys. Rev. D 98, 092001 (2018).

[78] O. Adriani et al. (L3 Collaboration), Phys. Lett. B 292, 472 (1992).

[79] E. M. Riordan et al., Phys. Rev. Lett. 59, 755 (1987).

[80] J. D. Bjorken, S. Ecklund, W. R. Nelson, A. Abashian, C. Church, B. Lu, L. W. Mo, T. A. Nunamaker, and P. Rassmann, Phys. Rev. D 38, 3375 (1988).

[81] J. Blümlein and J. Brunner, Phys. Lett. B 731, 320 (2014).

[82] S. Benson and A. Puig Navarro, Report No. LHCb-PUB2018-006, https://cds.cern.ch/record/2314368?ln=es.
[83] G. Aad et al. (ATLAS Collaboration), J. High Energy Phys. 01 (2013) 086.

[84] CMS Collaboration, Report No. CMS-PAS-HIG-12-015, https://cds.cern.ch/record/1460419?ln=en.

[85] S. Chatrchyan et al. (CMS Collaboration), Phys. Rev. Lett. 108, 111801 (2012).

[86] G. Aad et al. (ATLAS Collaboration), Phys. Lett. B 710, 538 (2012).

[87] G. Aad et al. (ATLAS Collaboration), Phys. Rev. Lett. 113, 171801 (2014).

[88] CMS Collaboration, Report No. CMS-PAS-HIG-14-037, https://cds.cern.ch/record/2063739? ln=en.

[89] L. Di Luzio, F. Mescia, and E. Nardi, Phys. Rev. D 96, 075003 (2017).

[90] J. P. Lees et al. (BABAR Collaboration), Phys. Rev. Lett. 107, 221803 (2011).

[91] A. M. Sirunyan et al. (CMS Collaboration), Eur. Phys. J. C 78, 789 (2018).

[92] Signals sensitive to the ALP-top coupling have been briefly discussed in Ref. [31] in the context of the nonlinear operator $\mathcal{A}_{2 D}(h)$. Nevertheless, the bound obtained followed from $a Z h$ interactions contained in $\mathcal{A}_{2 D}(h)$, which do not apply for $\mathcal{O}_{a \Phi}$ (i.e., in the linear EFT expansion at NLO).

[93] A. M. Sirunyan et al. (CMS Collaboration), J. High Energy Phys. 09 (2018) 148. 